\title{
Desempenho de clones de seringueira da série IAC 300 na região do planalto de São Paulo(1)
}

\author{
Paulo de Souza Gonçalves ${ }^{(2)}$, Antonio Lúcio Mello Martins ${ }^{(3)}$, Edson Luiz Furtado(4), Rosana Sambugaro(5), \\ Emma Luize Ottati(6), Altino Aldo Ortolani ${ }^{(7)}$ e Gentil Godoy Júnior ${ }^{(8)}$
}

\begin{abstract}
Resumo - O objetivo deste trabalho foi selecionar clones de seringueira (Hevea spp.) promissores para a região do planalto do Estado de São Paulo. De uma população de 70 clones, de polinização controlada, integrantes do experimento de avaliação em pequena escala, foram avaliados 16 da série IAC 300. $\mathrm{O}$ experimento foi instalado na Estação Experimental de Pindorama, no espaçamento de 7 x $3 \mathrm{~m}$, em delineamento de blocos casualizados, com três repetições, tendo os clones RRIM 600 e GT 1 como testemunhas. Quanto à produção de borracha seca, destacaram-se oito e dez clones superiores ao RRIM 600 e GT 1, respectivamente. Todos os clones avaliados apresentaram-se vigorosos em relação ao perímetro do caule na abertura do painel. Sete deles mostraram alta resistência; sete, resistência moderada, e dois, suscetíveis à antracnose do painel. Sugere-se sua avaliação em experimentos de grande escala, para avaliar, além da produção, os demais caracteres secundários, em diferentes ambientes, para futuras recomendações em larga escala para o Estado de São Paulo.
\end{abstract}

Termos para indexação: Hevea brasiliensis, casca, espessura, antracnose, rendimento.

\section{Performance of IAC 300 rubber clones in the plateau of São Paulo State, Brazil}

\begin{abstract}
The present paper shows the results of the selection of IAC 300 serial promising clones of rubber tree (Hevea spp.) for the plateau region of São Paulo State, Brazil. Eighteen clones were selected from a population of 70 clones resulted of controlled pollination, all evaluated in a small scale trial. The trial was laid out in randomized block design with three replications following the $7.0 \mathrm{~m} \times 3.0 \mathrm{~m}$ spacing at the Experimental Station of Pindorama. The clones RRIM 600 and GT 1 were used as control. Regarding to yield, eight and ten clones showed superiority to RRIM 600 and GT 1, respectively. All the selected clones showed to be vigorous regarding to the girth by the panel opening. Seven clones showed high resistance, seven with moderate resistance, and two were susceptible to the anthracnose panel canker. A complementary evaluation in large scale trials is to evaluate, besides yielding, other secondary characters, with the purpose of future recommendations in large scale for São Paulo State.
\end{abstract}

Index terms: Hevea brasiliensis, bark, thickness, anthracnosis, yields.

\section{Introdução}

A seringueira, pertencente ao gênero Hevea, da família Euphorbiaceae, tem a Hevea brasiliensis (Willd. ex Adr. de Juss.) Muell.-Arg. como a espécie

\footnotetext{
(1) Aceito para publicação em 9 de março de 2001 Parcialmente financiado pela Fapesp.

(2) Embrapa-IAC, Programa Integrado de São Paulo, Instituto Agronômico (IAC), Centro de Café e Plantas Tropicais, Programa Seringueira, Caixa Postal 28, CEP 13001-970 Campinas, SP. E-mail: paulog@cec.iac.br

(3)IAC, Estação Experimental de Agronomia de Pindorama, Caixa Postal 24, CEP 15830-000 Pindorama, SP.

E-mail: lmartins@zup.com.br

(4) Universidade Estadual Paulista, Fac. de Ciências Agronô-
}

mais importante do gênero. Na Ásia, ela é plantada como a principal fonte de borracha natural. Em 1999, mais de $68 \%$ da produção mundial foi originária da Tailândia, Indonésia e Malásia, que contribuíram, separadamente, com $32 \%, 25 \%$ e $11 \%$, respectiva-

micas, Caixa Postal 237, CEP 18603-970 Botucatu, SP E-mail: elfurtado@fca.unesp.br

(5) IAC, Centro de Café e Plantas Tropicais, Programa Seringueira. Bolsista da Fapesp. E-mail: rosambugaro@hotmail.com (6) IAC, Centro de Café e Plantas Tropicais, Programa Seringueira. E-mail: emminha@uol.com.br

(7) IAC, Centro de Ecofisiologia e Biofísica, Caixa Postal 28, CEP 13001-970 Campinas, SP. E-mail: altino@cec.iac.br

(8) IAC, Estação Experimental de Agronomia de Ubatuba, Caixa Postal 59, CEP 11680-000 Ubatuba, SP

E-mail: iac-uba@iconet.com.br 
mente, da produção mundial. A área total estimada de seringueira plantada é superior a 10 milhões de hectares. É tradicionalmente cultivada na região equatorial, situada entre $10^{\circ}$ Norte e $10^{\circ} \mathrm{Sul}$ do Equador.

O Brasil, embora seja o berço das espécies do gênero, contribuiu, no ano 2000 , com apenas $1 \%$ da produção mundial de 6.629 mil toneladas e consumiu $3 \%$ de um total de 7.361 mil toneladas da demanda mundial (International Rubber Study Group, 2001).

Há cerca de 120 anos, quando teve início sua domesticação, a seringueira foi considerada mais uma espécie selvagem da Amazônia. Durante esse período, o melhoramento genético contribui para seu desenvolvimento, elevando o nível de produção de $400 \mathrm{~kg} / \mathrm{ha} /$ ano para $3.000 \mathrm{~kg} / \mathrm{ha} /$ ano. Melhoristas têm tentado formular novas estratégias com o intuito de incrementar cada vez mais a produtividade do seringal, empregando um conjunto de métodos, ainda em desenvolvimento.

Segundo Gonçalves (1999), os objetivos do melhoramento da seringueira variam de acordo com as necessidades específicas de cada região, local ou país. No Estado de São Paulo, dois são os objetivos principais: o primeiro está voltado principalmente para o aumento da produção e vigor, como é normalmente praticado na região do Planalto; o segundo está relacionado com o aumento da produção e resistência ao mal-das-folhas, causado pelo Mycrocyclus ulei(P. Henn) v. Arx, na região do Litoral. A fim de atender às peculiaridades próprias dessas regiões, o programa levou em consideração o fato de que o Planalto Paulista, pelo menos até agora, não tem mostrado ataques epidêmicos do M. ulei, pois nessa região se verifica um período seco na época de reenfolhamento das plantas, optando-se, assim, pelos cruzamentos intraespecíficos, isto é, favoráveis à obtenção de materiais mais produtivos em látex; o litoral, ao contrário, por suas condições ecológicas favoráveis, condiciona o aparecimento de ataques severos daquele patógeno, e, diante disso, procurou-se dar ênfase aos cruzamentos interespecíficos, visando obter clones tolerantes. Por outro lado, os povoamentos de seringueira do Planalto Paulista vêm apresentando surtos de antracnose nas folhas e no painel de sangria (Furtado \& Silveira, 1990; Silveira et al., 1992). Entretanto, o objetivo do programa é principalmente a obtenção de clones com alto potencial de produção, seguida por outros caracteres secundários, cuja ausência pode causar a redução do vigor e da produtividade. Os principais caracteres secundários são: vigor, crescimento do caule durante o procedimento de sangria, espessura de casca virgem, boa regeneração da casca, resistência às principais doenças da região, tolerância à quebra pelo vento, e tolerância à seca do painel.

Este trabalho objetivou avaliar o potencial de produção, o vigor e outras variáveis de 16 clones de seringueira da série IAC 300.

\section{Material e Métodos}

Dezoito clones de seringueira, resultantes de polinizações controladas (Tabela 1), foram selecionados em Experimento de Avaliação de Clones em Pequena Escala (EAPE III), instalados na Estação Experimental de Agronomia de Pindorama, do Instituto Agronômico (IAC), de Pindorama, SP, a $21^{\circ} 73^{\prime}$ S de latitude, $48^{\circ} 56^{\prime} \mathrm{W}$ de longitude e 560 m de altitude, em solo Podzólico Vermelho-Amarelo TB, eutrófico, de textura média, profundo, abrupto e bem drenado (Lepsch \& Valadares, 1976).

Adotou-se o delineamento em blocos ao acaso, com três repetições, e cada parcela foi representada por seis árvores plantadas no espaçamento de 7,0 $\mathrm{m}$ entre linhas e 3,0 m entre plantas.

Na região, o clima é do tipo tropical continental, com predominância de verão úmido, níveis de energia típico dos trópicos, e um período de inverno seco, com temperatura e precipitações pluviais mais reduzidas. Em geral, o confronto entre as curvas mensais de evapotranspiração e de chuva resulta num equilíbrio hídrico favorável ao crescimento e à produção, de outubro a abril. As deficiências hídricas e os baixos níveis térmicos ocorrem de maio a setembro

Durante o período de crescimento dos clones, foram feitas mensurações anuais de perímetro do caule, a $0,50 \mathrm{~m}$ acima do calo de enxertia, no primeiro ano, e a 1,20 m, a partir do segundo ano. Dados de produção foram registrados a partir do sétimo ano de idade nas árvores da parcela que apresentaram perímetro do caule superior a $45 \mathrm{~cm}$. O registro da produção por clone foi efetuado pelo látex coagulado nas tigelas, coletado ao acaso, duas vezes ao mês, secado em condições normais de sombra e ventilação ao longo do período de avaliação, preso a cada árvore. O peso total anual de borracha por árvore/clone foi então dividido pelo número de coágulos, sendo o resultado expresso em gramas/árvore/sangria. A média geral do total árvores/clone foi expressa em gramas árvore/clone/ano. 
A partir desses dados estimou-se a produção por clone/hectare/ano, seguindo recomendações da Embrapa (1989). Adotou-se, no primeiro ano, sangria em d/2, com 140 sangrias por ano, e utilizaram-se, para cálculo, 240 árvores, $60 \%$ de um hectare de 400 árvores; e para o segundo ano, sangria em d/3, com 104 sangrias por ano, sendo utilizadas, para cálculo, 340 árvores, $85 \%$ de um hectare.

Nos dois anos de avaliação da produção foram utilizados diferentes sistemas de sangria. No primeiro ano, foi utilizado o sistema $1 / 2 \mathrm{~S} \mathrm{~d} / 25 \mathrm{~d} / 7.11 \mathrm{~m} / \mathrm{y}$ - sangria em meia espiral $(1 / 2 \mathrm{~S})$, realizada em intervalos de dois dias $(\mathrm{d} / 2)$, e a atividade de sangria em 5 dias na semana $(5 \mathrm{~d} / 7)$, durante 11 meses por ano $(11 \mathrm{~m} / \mathrm{y})$. No segundo ano, foi utilizado o sistema $1 / 2 \mathrm{~S} \mathrm{~d} / 35 \mathrm{~d} / 7.11 \mathrm{~m} / \mathrm{y}$.ET $2,5 \%$ $\mathrm{Pa} 10 / \mathrm{y}$ - sangria em meia espiral (1/2 S), em intervalos de três dias $(\mathrm{d} / 3)$, e a atividade de sangria, em 5 dias na semana $(5 \mathrm{~d} / 7)$, durante 11 meses por ano $(11 \mathrm{~m} / \mathrm{y})$, com estimulações feitas com ethefon a 2,5\% (ET 2,5\%), aplicado por pincelamento na canaleta de sangria, $(\mathrm{Pa}) \mathrm{dez}$ vezes ao ano $(10 / y)$

Amostras para determinação de espessura de casca virgem foram tomadas a 1,00 $\mathrm{m}$ de altura do caule de cada árvore, com auxílio de um vazador; em seguida, medidas com paquímetro; e o número de anéis de vasos laticíferos obtidos em laboratório, por meio das secções radiais longitudinais da casca.
Durante a execução do experimento, foram empregadas todas as práticas culturais convencionais do cultivo da seringueira (São Paulo, 1999), exceto o controle fitossanitário.

$\mathrm{Na}$ avaliação dos clones quanto à incidência da antracnose do painel, causada pelo fungo Colletotrichum gloesporioides (Penz.) Sacc. (Silveira et al., 1992), adotou-se, em todas as plantas, a contagem do número de cancros acima da superfície de corte, e aplicou-se uma escala de notas graduais, em função do número de cancros presentes: 1) cancros ausentes, até um cancro por painel; 2) um a dois cancros; 3 ) dois a três cancros; 4) três a quatro cancros; 5) cinco ou mais cancros, considerando-se como resistentes $(\mathrm{R})$ os clones nota 1 , moderadamente resistentes (MR) para as notas 2 e 3, e suscetíveis (S), para as notas 4 e 5 .

As observações obtidas nos 18 clones de seringueira incluíram, além do crescimento do caule e produção de borracha seca, em dois anos de sangria, porcentagem de plantas aptas à sangria, número de anéis de vasos laticíferos, espessura de casca virgem, conduzidos aos sete anos de idade, e, finalmente, avaliação da antracnose de painel. Esses clones, resultantes de uma primeira seleção, foram selecionados com base em suas produções de borracha e vigor. Para efeito de comparação, utilizaram-se como testemunhas os clones comerciais RRIM 600 e GT 1.

Tabela 1. Parentais dos clones da série IAC 300 e das testemunhas GT 1 e RRIM 600, avaliados na Estação Experimental de Agronomia de Pindorama, SP.

\begin{tabular}{ll}
\hline Clone & \\
\hline IAC 328 & RRIM 600 (Tjir 1 x PB 86) x PR 107 \\
IAC 329 & GT 711 x Tjir 16 \\
IAC 330 & RRIM 600 (Tjir 1 x PB 86) x GT 711 \\
IAC 331 & RRIM 600 (Tjir 1 x PB 86) x AVROS 1328 (AVROS 214 x AVROS 317) \\
IAC 332 & GT 711 x RRIM 600 (Tjir 1 x PB 86) \\
IAC 333 & C 228 x Tjir 16 \\
IAC 334 & Fx 25 (F 361 x AVROS 49) x AVROS 1126 \\
IAC 335 & GT 711 x RRIM 511 (Pil A 44 x Pil B 16) \\
IAC 336 & RRIM 600 (Tjir 1 x PB 86) x PR 107 \\
IAC 337 & RRIM 600 (Tjir 1 x PB 86) x PR 107 \\
IAC 338 & PB 5/63 (PB 56 x PB 24) x RRIM 512 (Pil B 84 x Pil A 44) \\
IAC 339 & RRIM 600 (Tjir 1 x PB 86) x RRIM 513 (Pil B 16 x Pil A 44) \\
IAC 340 & RRIM 600 (Tjir 1 x PB 86) x RRIM 513 (Pil B 16 x Pil A 44) \\
IAC 341 & GT 711 x PB 86 \\
IAC 342 & AVROS 363 x RRIM 527 (Pil B 50 x Pil B 84) \\
IAC 343 & AVROS 1518 (AVROS 214 x AVROS 256) x RRIM 527 (Pil B 50 x Pil B 84) \\
GT 1 & Clone primário \\
RRIM 600 & Tjir 1 x PB 86 \\
\hline
\end{tabular}

(1)IAC: Instituto Agronômico; GT: Gondang Tapen; RRIM: Rubber Research Institute of Malasia; PB: Prang Besar; F: Ford; Fx: Cruzamento Ford; Tjir Tjirandji; AVROS: Algemene Vereniging Rubber Planters Oostkust Sumatra; Pil: Pilmoor; PR: Proefstation voor Rubber; C: Cavala 


\section{Resultados e Discussão}

Foram detectadas diferenças significativas a $1 \%$ de probabilidade, na produção de borracha seca, dentro de cada ano, o que indica a existência de variabilidade dentro da população estudada. Não foi considerada a análise agrupada, uma vez que em cada ano foi utilizado um diferente sistema de sangria, optando-se, dessa forma, pela análise de variância individual (Tabela 2).

No segundo ano, sob o sistema de sangria $1 / 2 \mathrm{~S}$ $\mathrm{d} / 3$ 5d/7.11m/y.ET 2,5\% Pa 10/y, houve uma resposta na produção de todos os clones por causa da aplicação do ethefon a $2,5 \%$ no painel de sangria. O clone IAC 328 foi o que mais produziu, com $1.867 \mathrm{~kg} / \mathrm{ha} /$ ano de borracha seca, e aumentos de produção em torno de $100 \%$ em relação ao primeiro ano de produção.

A média dos dois anos de produção dos melhores clones, em relação à da testemunha RRIM 600, variou de $109 \%$ a $131 \%$ (Tabela 3 ). Em relação à testemunha GT 1, a média desses clones foi um pouco superior ( $103 \%$ a $148 \%$ ), considerando que o GT 1 apresentou produção inferior à do RRIM 600 (Gonçalves et al., 1993). O clone IAC 328 foi $31 \%$ superior ao RRIM 600, e 46\% superior ao GT 1 . A produção do IAC 331, do IAC 330 e do IAC 329 foi de $27 \%, 25 \%$ e $18 \%$, respectivamente, superior à do RRIM 600, e 40\%, 38\% e 30\%, respectivamente, superior à do GT 1 .

Tabela 2. Valores dos quadrados médios, coeficientes de variação $(\mathrm{CV})$ e média geral da produção ( $\mathrm{g}$ de borracha seca/sangria/árvore), referentes à análise de variância de 18 clones de seringueira, nos dois primeiros anos, na Estação Experimental de Agronomia de Pindorama, SP.

\begin{tabular}{lrcc}
\hline \multirow{2}{*}{ Fonte de variação } & \multirow{2}{*}{ GL } & \multicolumn{2}{c}{ Quadrado médio $^{(1)}$} \\
\cline { 3 - 4 } & & Ano 1 & Ano 2 \\
\hline Blocos & 2 & 54,9944 & 43,1223 \\
Clones & 17 & $117,5112^{* *}$ & $310,9065^{* *}$ \\
Resíduo & 34 & 23,5825 & 40,7807 \\
\hline CV (\%) & & 17,51 & 16,65 \\
Média geral & & 27,74 & 38,35 \\
\hline
\end{tabular}

(1)Ano 1: sistema de sangria $1 / 2 \mathrm{~S} \mathrm{~d} / 25 \mathrm{~d} / 7.11 \mathrm{~m} / \mathrm{y}$; Ano 2: sistema de sangria $1 / 2 \mathrm{~S} \mathrm{~d} / 35 \mathrm{~d} / 7.11 \mathrm{~m} / \mathrm{y}$. ET 2,5\% Pa 10/y. ** Significativo a $1 \%$ de probabilidade.
Ocorreråm diferenças significativas $(\mathrm{P}<0,01)$ entre clones, em relação ao incremento do perímetro do caule (Tabela 4). A presença de interação entre clones e anos $(p<0,01)$ revela que os clones apresentaram diferentes incrementos nos diferentes anos. Segundo Gonçalves et al. (1993), o desenvolvimento dos clones no período adulto é um caráter muito importante para a manutenção da constância de produção, e que, por sua vez, contribuirá para a redução de quebra de árvores pelo vento. Em geral, os mesmos autores enfatizam que os incrementos são significativamente menores na maturidade comparado com o de imaturidade, indicando que o crescimento das árvores nesses clones é afetado pela explotação do látex.

O maior incremento médio anual do perímetro do caule relativo ao período de imaturidade foi apresentado pelo clone IAC 334, seguido pelos clones IAC 340, IAC 328 e IAC 342 (Tabela 5). O rápido crescimento significa redução do período de imaturidade o que torna esses clones aptos à abertura do painel aos seis anos de idade. No período adulto, ou seja, no período de sangria, considerando que o crescimento dos clones nessa fase é muito importante para a manutenção da constância de produção, somente os clones IAC 342, IAC 340, GT 1 (testemunha) e IAC 341 se destacaram. Nessa fase, segundo Castro (1990), há uma competição entre o dreno induzido pela sangria e os processos de crescimento, capazes de afetar adversamente o potencial de produtividade. Porém, existem clones que apresentam alta capacidade de produção e alto incremento anual do caule e mantêm produção constante durante a fase adulta (Abraham \& Tayler, 1967).

A média do perímetro do caule na abertura dos painéis está relacionada com o porcentual de plantas aptas à sangria, o que pode ser observado nos clones IAC 334, IAC 340 e IAC 342. Mesmo tendo em conta a variabilidade entre as médias de perímetro dos clones, a média geral de 41,62 indica que o desenvolvimento das árvores foi inferior ao desempenho dos clones em Votuporanga (região do Planalto Paulista), que no sétimo ano alcançou a média de 45,35 cm (Gonçalves et al., 1999).

Os valores médios de anéis de vasos laticíferos por clone encontrados variaram de 11,00 (IAC 343) a 30,50 (IAC 331) (Tabela 6). Segundo Webster \& 
Tabela 3. Estimativas de produção de borracha seca e porcentagem de ganho, em relação às testemunhas (RRIM 600 e GT 1) referentes, e aos dois anos de sangria de 18 clones selecionados no Experimento de Avaliação em Pequena Escala, instalado na Estação Experimental de Agronomia de Pindorama, SP(1).

\begin{tabular}{|c|c|c|c|c|c|c|c|c|}
\hline \multirow[t]{2}{*}{ Clone } & \multicolumn{2}{|c|}{ Ano $1^{(2)}$} & \multicolumn{2}{|c|}{ Ano $2^{(3)}$} & \multicolumn{2}{|c|}{ Média } & \multicolumn{2}{|c|}{$\begin{array}{l}\text { Porcentagem em relação às } \\
\text { testemunhas }\end{array}$} \\
\hline & $\mathrm{g} /$ sangria/árvore & $\mathrm{kg} / \mathrm{ha} / \mathrm{ano}$ & g/sangria/árvore & $\mathrm{kg} / \mathrm{ha} / \mathrm{ano}$ & g/sangria/árvore & $\mathrm{kg} / \mathrm{ha} / \mathrm{ano}$ & RRIM 600 & GT 1 \\
\hline IAC 328 & $37,57 \mathrm{a}$ & 938 & $52,81 \mathrm{a}$ & 1.867 & 45,19 & 1.403 & 131 & 146 \\
\hline IAC 329 & $36,17 \mathrm{ab}$ & 902 & 47,28abcde & 1.672 & 41,73 & 1.287 & 118 & 130 \\
\hline IAC 330 & $35,80 \mathrm{a}$ & 896 & $50,18 \mathrm{abc}$ & 1.774 & 43,02 & 1.335 & 125 & 138 \\
\hline IAC 331 & $35,17 \mathrm{a}$ & 878 & $50,85 \mathrm{ab}$ & 1.798 & 43,01 & 1.338 & 127 & 140 \\
\hline IAC 332 & $35,00 \mathrm{a}$ & 873 & $45,54 \mathrm{abcde}$ & 1.610 & 40,27 & 1.242 & 113 & 126 \\
\hline IAC 333 & $35,00 \mathrm{abc}$ & 874 & 44,17abcde & 1.562 & 39,59 & 1.218 & 110 & 122 \\
\hline IAC 334 & $31,00 \mathrm{abcd}$ & 774 & $50,23 \mathrm{abcd}$ & 1.776 & 40,62 & 1.275 & 125 & 138 \\
\hline IAC 335 & $29,86 a b c d$ & 745 & 43,95abcde & 1.554 & 36,91 & 1.150 & 109 & 121 \\
\hline IAC 336 & $28,57 \mathrm{abcd}$ & 713 & 37,37abcdefg & 1.321 & 32,97 & 1.017 & 93 & 103 \\
\hline IAC 337 & 27,33abcd & 682 & $32,58 \mathrm{bcdefg}$ & 1.152 & 29,96 & 917 & 81 & 90 \\
\hline IAC 338 & $25,50 \mathrm{abcd}$ & 636 & $28,05 \mathrm{efg}$ & 992 & 26,78 & 814 & 70 & 77 \\
\hline IAC 339 & $25,25 \mathrm{abcd}$ & 630 & $30,61 \mathrm{defg}$ & 1.082 & 27,93 & 856 & 76 & 84 \\
\hline IAC 340 & $19,92 \mathrm{bcd}$ & 497 & $31,72 \mathrm{cdefg}$ & 1.122 & 25,82 & 809 & 79 & 87 \\
\hline IAC 341 & $19,50 \mathrm{~d}$ & 487 & $23,56 f g$ & 833 & 21,53 & 660 & 59 & 65 \\
\hline IAC 342 & $19,83 \mathrm{bcd}$ & 495 & $28,37 \mathrm{efg}$ & 1.003 & 24,10 & 749 & 71 & 78 \\
\hline IAC 343 & $19,17 \mathrm{~cd}$ & 478 & $21,44 \mathrm{~g}$ & 758 & 20,31 & 618 & 53 & 59 \\
\hline RRIM 600 & 26,39abcd & 659 & 40,17 abcdef & 1.420 & 33,28 & 1.040 & 100 & 111 \\
\hline GT 1 & 31,10abcd & 776 & $36,28 \mathrm{abcdefg}$ & 1.283 & 33,69 & 1.029 & 90 & 100 \\
\hline
\end{tabular}


Paardekooper (1989), o número de anéis de vasos laticíferos é o fator, inerente ao clone, mais importante do sistema laticífero que aumenta com a idade da árvore.

A maioria dos clones selecionados apresentaram vigor, avaliado pelo perímetro do caule, espessura de casca virgem e número de anéis de vasos laticíferos superiores ao do clone-testemunha RRIM 600. Esses caracteres secundários são considerados importantes numa primeira seleção, por influir na produtividade do clone (Gonçalves et al., 1995).

O período de avaliação do experimento pode ser considerado satisfatório, uma vez que o período de avaliação e seleção é estimado em cerca de 25 a 30 anos (Clément-Demange et al., 1995; Gonçalves et al., 1997). Os dados de produção e vigor apresentados neste trabalho são resultados preliminares dos clones em uma primeira seleção com vistas a testá-los em experimentos de avaliação de grande escala a serem instalados em várias regiões do Estado de São Paulo. A seleção deve basear-se nas correlações obtidas no primeiro ano de produção com os anos subseqüentes (Alika, 1980; Ong, 1980; Marques \& Gonçalves, 1990). Estes autores observaram que é possível utilizar os clones que apresentaram potencial de produção nos primeiros anos de avaliação e incluílos em experimentos de avaliação do tipo Grande Escala com sucesso.

Tabela 4. Valores dos quadrados médios da análise de variância, coeficientes de variação $(\mathrm{CV})$ e média geral do incremento anual do perímetro do caule, em centímetros, referente aos nove anos de avaliação em 18 clones de seringueira, na Estação Experimental de Agronomia de Pindorama, SP.

\begin{tabular}{lrc}
\hline Fonte de variação & GL & $\begin{array}{c}\text { Incremento anual } \\
\text { do caule }\end{array}$ \\
\hline Blocos & 2 & 13,5266 \\
Clones & 17 & $9,1706^{* *}$ \\
Resíduo (clones) & 34 & 1,8238 \\
Anos & 8 & $182,4797^{* *}$ \\
Clones x anos & 136 & $4,3450^{* *}$ \\
Anos x blocos & 16 & $3,9269^{* *}$ \\
Resíduo (anos) & 272 & 2,0876 \\
\hline CV (\%) (clones) & & 8,43 \\
CV (\%) (anos) & & 27,07 \\
Média geral (cm) & 5,34 \\
\hline **Significativo a 1\% de probabilidade &
\end{tabular}

**Significativo a $1 \%$ de probabilidade.

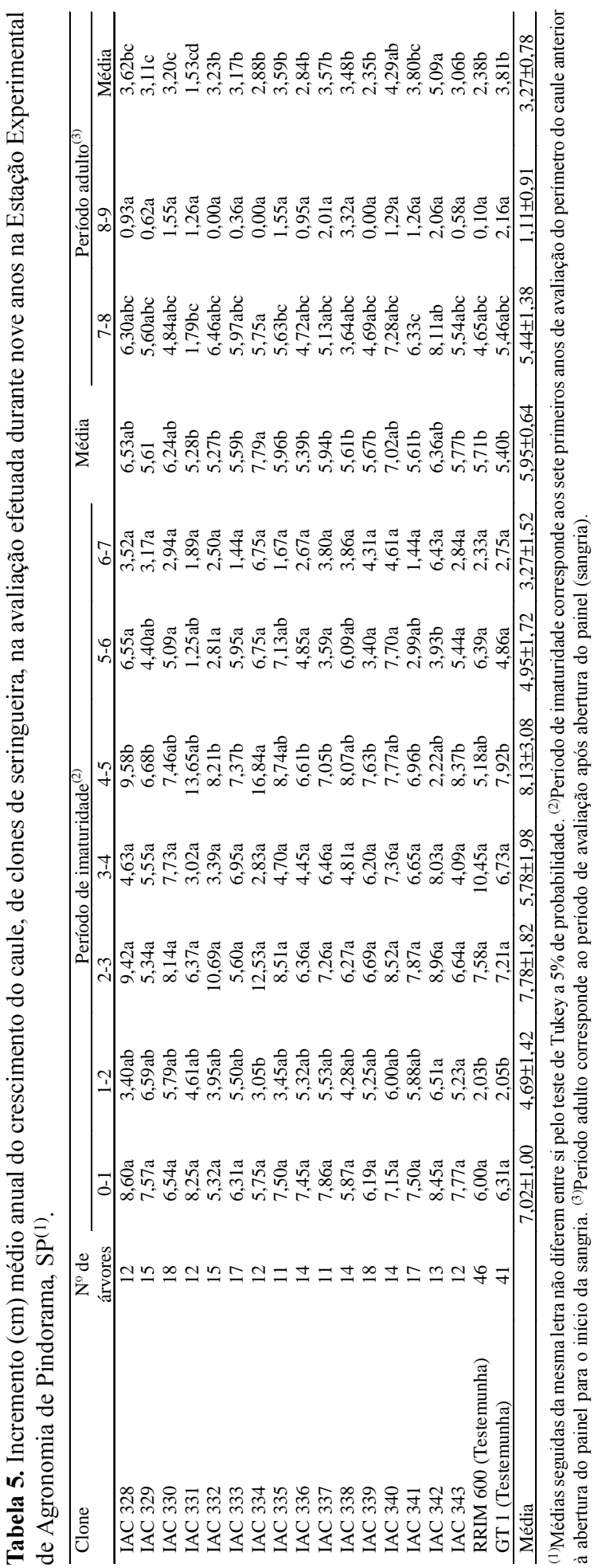


Tabela 6. Parâmetros avaliados na ocasião da abertura do painel de 18 clones de seringueira, na Estação Experimental de Agronomia de Pindorama, SP.

\begin{tabular}{|c|c|c|c|c|c|c|}
\hline Clone & $\begin{array}{l}\mathrm{N}^{\mathrm{o}} \mathrm{de} \\
\text { plantas }\end{array}$ & $\begin{array}{l}\text { Perímetro } \\
\text { médio do } \\
\text { caule }(\mathrm{cm})\end{array}$ & $\begin{array}{l}\text { Plantas aptas } \\
\text { à sangria } \\
(\%)^{(1)}\end{array}$ & $\begin{array}{l}\text { № médio de } \\
\text { anéis de } \\
\text { vasos } \\
\text { laticíferos }\end{array}$ & $\begin{array}{c}\text { Espessura } \\
\text { média de casca } \\
\text { virgem }(\mathrm{mm})\end{array}$ & $\begin{array}{l}\text { Antracnose } \\
\text { de painel }\end{array}$ \\
\hline IAC 328 & 12 & 45,70 & 60 & 17,00 & 5,26 & 2 \\
\hline IAC 329 & 15 & 39,30 & 40 & 18,00 & 5,80 & 2 \\
\hline IAC 330 & 18 & 43,69 & 72 & 18,57 & 5,88 & 1 \\
\hline IAC 331 & 12 & 39,04 & 50 & 30,50 & 7,18 & 1 \\
\hline IAC 332 & 15 & 36,87 & 27 & 18,00 & 5,27 & 2 \\
\hline IAC 333 & 17 & 39,12 & 71 & 19,50 & 5,99 & 1 \\
\hline IAC 334 & 12 & 54,50 & 100 & 17,67 & 5,78 & n.a. \\
\hline IAC 335 & 11 & 41,70 & 82 & 15,50 & 5,15 & 2 \\
\hline IAC 336 & 14 & 37,71 & 50 & - & - & 1 \\
\hline IAC 337 & 11 & 41,55 & 55 & 21,00 & 6,08 & 2 \\
\hline IAC 338 & 14 & 39,25 & 43 & - & - & 1 \\
\hline IAC 339 & 18 & 39,67 & 44 & 15,00 & 5,52 & 1 \\
\hline IAC 340 & 14 & 49,11 & 93 & 16,00 & 5,26 & 5 \\
\hline IAC 341 & 17 & 39,29 & 59 & 13,25 & 4,32 & 2 \\
\hline IAC 342 & 13 & 44,53 & 92 & 12,25 & 5,15 & 3 \\
\hline IAC 343 & 12 & 40,38 & 50 & 11,00 & 3,42 & 1 \\
\hline RRIM 600 (testemunha) & 41 & 39,96 & 56 & 16,00 & 5,59 & 2 \\
\hline GT 1 (testemunha) & 46 & 37,83 & 65 & 17,50 & 5,27 & 4 \\
\hline Média & 17 & $41,62 \pm 4,47$ & $62 \pm 20,02$ & $17,30 \pm 4,39$ & $5,43 \pm 0,81$ & \\
\hline
\end{tabular}

(1) Árvores com perímetro superior a $45 \mathrm{~cm}$ a $1,20 \mathrm{~m}$ de altura do calo de enxertia; a abertura do painel foi realizada no sétimo ano de desenvolvimento vegetativo. ${ }^{(2)}$ Número médio de lesões por painel: 1) 0 a 1 cancro (resistentes); 2) 1 a 2 cancros (moderadamente resistentes); 3) 2 a 3 cancros (moderadamente resistentes); 4) 4 a 5 cancros (suscetíveis); 5) 5 ou mais cancros/planta (suscetíveis); n.a: não avaliado.

A incidência de antracnose do painel, causada pelo fungo Colletotrichum gloeosporioides Penz., segundo Silveira et al. (1992), ocorre no painel de sangria, no Estado de São Paulo em períodos favoráveis. Coincidindo com os períodos do ano mais frios e com umidade elevada, o patógeno provoca lesões em grandes porções da casca em recuperação. Apresentaram alta resistência a esse fungo os clones IAC 330, IAC 331, IAC 333, IAC 336, IAC 338, IAC 339 e IAC 343; moderada resistência, os clones IAC 328, IAC 329, IAC 332, IAC 335, IAC 337, IAC 341 e GT 1; os clones IAC 340 e RRIM 600 foram suscetíveis.

\section{Conclusões}

1. Os clones IAC 328, IAC 329 e IAC 330 apresentam melhor desempenho de produção; recomenda-se seu plantio em pequena escala na região de São José do Rio Preto.
2. Quanto ao perímetro e incremento do caule, os clones IAC 334 e IAC 340 apresentam melhor desempenho; sugere-se o seu uso como parentais em futuros programas de melhoramentos.

3. Os clones IAC 331 e IAC 337 mostram maior espessura de casca e maior número de anéis de vasos laticíferos.

4. Os clones IAC 330, IAC 331, IAC 333, IAC 336, IAC 338, IAC 339 e IAC 343 apresentam alta resistência à antracnose do painel.

\section{Agradecimentos}

Às laboratoristas Andréa Cardoso Guerreiro e Lígia Regina Lima Gouvêa, pelas análises do sistema laticífero contido na casca da seringueira; à Graziela dos Santos Lima, pela preparação gráfica; aos técnicos agrícolas Edson Bernardes de Freitas e Ari de Camargo, do Núcleo Experimental de Campi- 
nas, do Programa Seringueira do Centro de Café e Plantas Tropicais, pela colaboração na coleta dos dados de campo.

\section{Referências}

ABRAHAM, P. D.; TAYLER, R. S. Tapping of Hevea brasiliensis. Tropical Agriculture, St. Augustine, v. 44, p. $15,1967$.

ALIKA, J. E. Possibilities of early selection in Hevea brasiliensis: short note. Silvae Genetica, Frankfurt, v. 29, n. 3/4, p. 161-162, 1980.

CASTRO, P. R. C. Bases fisiológicas da produção do látex e da estimulação de Hevea brasiliensis Muel. Arg. In: BERNARDES, M. S. (Ed.). Sangria da seringueira. Piracicaba: Esalq, 1990. p. 122.

CLÉMENT-DEMANGE, A.; NICOLAS, D.; LEGNATE, H.; RIVANO, F.; LE GUEN, V.; GNAGNE, M.; CHAPUSET, T. Hevea strategies de selection. Plantations, Recherche, Développement, Montpellier, v. 2, n. 3 , p. 5-19, 1995.

EMBRAPA. Centro Nacional de Pesquisa de Seringueira e Dendê (Manaus, AM). Melhoramento genético da seringueira. Manaus, 1989. 23 p. (Documentos, 10).

FURTADO, E. L.; SILVEIRA, A. P. Doenças do painel de sangria da seringueira. In: BERNARDES, M. S. (Ed.). Sangria da seringueira. Piracicaba: Esalq, 1990. p. 111-125.

GONÇALVES, P. de S. Recomendação de clones de seringueira para o Estado de São Paulo. In: CICLO DE PALESTRAS SOBRE A HEVEICULTURA PAULISTA, 1 . 1998, Barretos. Anais... Barretos: Secretaria de Agricultura e Abastecimento/Associação Paulista de Produtores e Beneficiadores de Borracha, 1999. p. 115-140.

GONÇALVES, P. de S.; BORTOLETTO, N.; ORTOLANI, A. A.; BELLETTI, G. O.; SANTOS, W. R. dos. Desempenho de novos clones de seringueira. III. Seleções promissoras para a região de Votuporanga, Estado de São Paulo. Pesquisa Agropecuária Brasileira, Brasília, v. 34, n. 6, p. 971-980, jun. 1999.

GONÇALVES, P. de S.; CARDOSO, M.; MENTE, E. M.; MARTINS, A. L. M.; GOTTARDI, M. V. C. Desempenho preliminar de clones de seringueira na região de São José do Rio Preto, planalto do Estado de São Paulo. Bragantia, Campinas, v. 52, n. 2, p. 119-130, 1993.

GONÇALVES, P. de S.; MARTINS, A. L. M.; BORTOLETTO, N.; CARVALHO, A. Z. C. Relationship among yield, girth and some structural characters of the laticiferous system in young seedlings of rubber trees (Hevea). Brazilian Journal of Genetics, Ribeirão Preto, v. 18 , n. 3, p. 421-428, 1995.

GONÇALVES, P. de S.; ORTOLANI, A. A.; CARDOSO, $M$. Melhoramento genético da seringueira: uma revisão. Campinas: Instituto Agronômico, 1997. 55 p. (Documento IAC, 54).

INTERNATIONAL RUBBER STUDY GROUP (Wembley, Inglaterra). Production of natural rubber: Table 7. Rubber Statistical Bulletin, Wembley, v. 55, n. 11, p. 9-10, 2001.

LEPSCH, I. F.; VALADARES, J. M. da S. Levantamento pedológico detalhado da Estação Experimental de Pindorama, SP. Bragantia, Campinas, v. 35, n. 1, p. 13-40, 1976.

MARQUES, J. R. B.; GONÇALVES, P. de S. Testes precoces de produção na seleção de plantas de seringueira. Pesquisa Agropecuária Brasileira, Brasília, v. 25, n. 7, p. 1065-1077, jul. 1990 .

ONG, S. H. Correlations between yield girth and bark thickness of RRIM clones trials. Journal of the Rubber Research Institute of Malaysia, Kuala Lumpur, v. 29, n. 1, p. 1-14, 1980.

SÃO PAULO (Estado). Secretaria de Agricultura e Abastecimento. Comissão Técnica da Seringueira. A cultura da seringueira para o Estado de São Paulo. Campinas: CATI, 1999. 91 p. (Manual, 72).

SILVEIRA, A. P.; FURTADO, E. L.; LOPES, M. E. B. M. Antracnose: nova doença do painel de sangria da seringueira. Summa Phytopathologica, Piracicaba, v. 18, n. 3/4, p. 195-200, 1992.

WEBSTER, C. C.; PAARDEKOOPER, E. C. The botany of the rubber tree. In: WEBSTER, C. C.; BAULKWILL, W. J. (Ed.). Rubber. New York: Longman, 1989. p. $57-84$. 